\title{
Mortality and survival in myasthenia gravis: a Danish population based study
}

P B Christensen, T S Jensen, I Tsiropoulos, T Sørensen, M Kjær, E Højer-Pedersen, M J K Rasmussen, E Lehfeldt

\begin{abstract}
Objectives-To study mortality and survival of patients with myasthenia gravis. Methods-290 patients with myasthenia gravis were studied, including 212 incident cases identified during a comprehensive epidemiological study of myasthenia gravis in western Denmark 1975-89. Follow up was performed on 31 December 1994. Survival curves were constructed using the life table method. Patient data were compared with data from the public Danish population statistics. Death certificates were provided from the National Registry of Death.
\end{abstract}

Results-The annual average crude mortality rate was 1.8 per million (range 1.5-2.2). The myasthenia gravis related mortality rate (myasthenia gravis as underlying or contributory cause) was 1.4 per million (range 1.1-1.8). The age specific mortality rates were low below 50 years. After this age the mortality increased with age in both sexes; after 60 years more rapidly in men than in women. The overall survival rates three, five, 10 , and 20 years from diagnosis were $85 \%, 81 \%, 69 \%$, and $63 \%$ respectively. The survival of both sexes was shorter than that of the corresponding Danish population. Old age at diagnosis, a classification in OssermanGenkins group IIB or III, and the presence of a thymoma were associated with a less favourable prognosis. The three, five, 10 , and 20 year survival rates of thymectomised patients were $94 \%, 94 \%, 86 \%$, and $79 \%$ respectively. The corresponding figures for the non-thymectomised patients were $78 \%, 71 \%, 56 \%$, and $51 \%$. A Cox regression analysis showed that this apparently significant effect of thymectomy was because the thymectomised patients were younger than the non-thymectomised patients. Furthermore, at the time of diagnosis of myasthenia gravis the non-thymectomised patients had a higher frequency of serious conditions associated with myasthenia gravis than the thymectomised patients.

Conclusion-Patients with myasthenia gravis generally have a relative good prognosis although their survival is shorter than that of the corresponding population. Old age, a classification in OssermanGenkins group III, and the presence of a thymoma are associated with a less favourable prognosis. In this study, the apparently significant effect of thymec- tomy was because the thymectomised patients were younger than nonthymectomised patients and because the non-thymctomised patients had a higher frequency of serious conditions associated with myasthenia gravis.

\section{(f Neurol Neurosurg Psychiatry 1998;64:78-83)}

Keywords: myasthenia gravis; mortality; survival; thymectomy

Myasthenia gravis is an autoimmune disease characterised by weakness of voluntary muscles due to the binding of antibodies to acetylcholine receptors on the muscle membrane..$^{1-3}$ The disease varies from mild cases with purely ocular symptoms to severe cases with generalised weakness and respiratory insufficiency. Most descriptions of the prognosis of myasthenia gravis are based on qualitative data obtained by an assessment of the clinical course using the Osserman-Genkins classification $^{4}$ and a disability scale. ${ }^{5}$ A more detailed description of possible prognostic factors in myasthenia gravis has not previously been provided using population based data. It is at present unclear if thymectomy improves survival and to what extent basic clinical data are correlated with survival. This study presents mortality and survival data of patients with myasthenia gravis diagnosed in western Denmark during the period 1975-89. We were particularly interested in analysing how mortality and survival of patients with myasthenia gravis were influenced by age, sex, clinical type of myasthenia gravis, and thymectomy.

\section{Methods}

The study involved all hospital departments, both neurological and non-neurological, serving the population of western Denmark. The study population was 2.8 million, about half of the Danish population. The age and sex distribution of the study population did not differ from that of the entire country. The case finding procedure, incidence, and prevalence data have previously been described in detail. ${ }^{6}$ In brief, we reviewed 412 records coded and diagnosed under the diagnostic category 733 myasthenia gravis (8th revision of the International Classification of Diseases (ICD)) in the period from 1 January 1975 to 31 December 1989 . We excluded 113 cases (81 women, 32 men) with various diagnoses (polyneuropathy, myopathy, multiple sclerosis, neurasthenic 
syndrome), including five cases of EatonLambert myasthenic syndrome.

In total, 299 cases fulfilled the following criteria for a clinical diagnosis of myasthenia gravis: (1) muscle weakness and rapid fatigue in one or more muscle groups; (2) muscle weakness aggravated by exercise and relieved by rest; and (3) a significant response to anticholinesterase drugs. Patients with a doubtful or negative anticholinesterase response were included if they otherwise fulfilled the diagnostic criteria and if the subsequent course was compatible with myasthenia gravis. Furthermore, if diagnosed within recent years such patients were only included if they had a significantly raised antiacetylcholine receptor (anti-AChR) antibody titre.

Of the 299 patients, we excluded seven with penicillamine induced myasthenia gravis and two with transient neonatal myasthenia gravis. The remaining 290 patients entered the mortality study as prevalent cases. The 212 incident cases diagnosed during the period from 1975 to 1989 entered the survival study. According to the Osserman-Genkins classification, these 212 cases were grouped as follows: group I (ocular myasthenia gravis): 23 cases $(11 \%)$; group IIA (mild generalised myasthenia gravis): 92 cases (43\%); group IIB (moderate generalised myasthenia gravis): 44 cases (21\%); group III (acute fulminating myasthenia gravis): 29 cases (14\%); and group IV (late severe myasthenia gravis): six cases $(3 \%)$. Eighteen cases $(8 \%)$ could not be classified. Most of these patients had a course of disease that was dominated by severe bulbar symptoms.

The diagnosis of myasthenia gravis was supported by (1) a significantly raised anti-AChR antibody titre in 163 of the 183 patients tested $(89 \%)$; (2) decrement on repetitive nerve stimulation in 100 of 136 patients (74\%) examined; and (3) a positive edrophonium chloride (tensilon) test in all except three patients. One hundred and fifty one patients fulfilled at least two of the three criteria.

Of the 212 incident cases 94 (44\%) underwent thymectomy. A thymoma was present in 26 patients ( $12 \%$ of the incident cases).

At the follow up on 31 December 1994, all patients were traced through the computerised medical index. Death certificates were provided from The National Registry of Death in which all deaths in Denmark are registered.

Because of the uncertainty in defining onset of disease, we preferred the more clearly defined time of diagnosis as the zero point in all calculations. The average duration of the follow up period from the time of diagnosis in the 212 incident cases was 9.2 (range 1-20) years.

\section{STATISTICS}

Confidence intervals of the mortality rates were calculated using the Poisson distribution. A univariate survival analysis for the different clinical variables was performed using the life table method and the log rank test. ${ }^{7}$ Confidence intervals of the survival rates were calculated by means of the Greenwood estimate. ${ }^{8} \mathrm{~A}$ multivariate Cox regression analysis ${ }^{9}$ was used
Table 1 Underlying causes of death in patients with myasthenia gravis ( $n=100$ deaths)

\begin{tabular}{ll}
\hline & Deaths $(n)$ \\
\hline Cardiovascular disease & 31 \\
Myasthenia gravis & 27 \\
Malignant disease` & 15 \\
Pneumonia & 4 \\
Other pulmonary disease & 7 \\
Cerebrovascular disease & 6 \\
Perforated diverticulitis $\dagger$ & 3 \\
Perforated gastric ulcer & 2 \\
Urosepsis $\$$ & 1 \\
Accident & 2 \\
Unknown & 2 \\
\hline
\end{tabular}

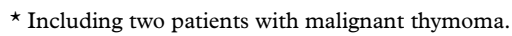

† All patients treated with steroids.

$\ddagger$ One patient treated with steroids.

$\checkmark$ Treated with steroids.

to analyse which clinical variables had independent effects on survival. The difference between two averages was estimated by the $\chi^{2}$ test. For all tests, $\mathrm{p}$ values $<0.05$ were considered significant. To include matched population statistics in the survival analysis, we employed a computer program developed at The Danish Institute for Clinical Epidemiology (provided by Dr N Kock-Henriksen). The program is based on data from the public population statistics (Danmarks Statistik).

\section{Results}

\section{MORTALITY}

One hundred of the 290 identified cases of myasthenia gravis died during the study period. Table 1 shows the underlying causes of death according to the death certificates. The most common cause was cardiovascular disease in 31 cases (31\%). Myasthenia gravis was mentioned as an underlying cause in 27 cases $(27 \%)$. In two cases a myasthenic crisis was mentioned specifically on the death certificate. In 52 cases myasthenia gravis was a contributory cause of death. In 21 cases there were no indications of myasthenia gravis on the death certficate. During long term steroid treatment five patients died (perforated diverticulitis in three, urosepsis in one, and perforated gastric ulcer in one).

The annual average crude mortality rate was 1.8 per million (range 1.5-2.2); in women 1.6 (range 1.1-2.1), in men 2.0 (range 1.5-2.6). The myasthenia gravis mortality rate, defined as myasthenia gravis as either an underlying or a contributory cause of death, was 1.4 per million (range 1.1-1.8).

Table 2 shows the age and sex specific mortality rates. After 50 years the mortality rates increased with age; after 60 years more rapidly in men than in women.

The average annual crude mortality rate increased during the observation period: 1975-9: 0.7 per million; 1980-4: 1.6, and 1985-9: 2.5 . The average annual case:fatality ratio was constant: 1975-9: 0.02, 1980-4: 0.03, and 1985-9: 0.03.

SURVIVAL

Sixty four of the 212 incident cases died during the observation period. The survival rates for all patients three, five, 10 , and 20 years from the time of diagnosis were $85 \%, 81 \%, 69 \%$ and 
Table 2 Myasthenia gravis, western Denmark (sex and age specific mortality rates per million per year (95\% CIs)

\begin{tabular}{lccc}
\hline Age group $(y)$ & Women & Men & Total \\
\hline $20-29$ & $0.24(0.01-1.34)$ & 0 & $0.12(0.003-0.67)$ \\
$30-39$ & $0.23(0.01-1.28)$ & 0 & $0.11(0.003-0.61)$ \\
$40-49$ & $0.87(0.18-2.54)$ & $0.29(0.01-1.62)$ & $0.58(0.16-1.48)$ \\
$50-59$ & $1.64(0.52-3.82)$ & $1.68(0.54-3.91)$ & $1.66(0.80-3.05)$ \\
$60-69$ & $2.90(1.25-5.71)$ & $5.59(3.31-9.22)$ & $4.22(2.70-6.20)$ \\
$70-79$ & $6.52(3.65-10.76)$ & $11.06(6.75-17.14)$ & $8.51(5.96-11.83)$ \\
$\geqslant 80$ & $11.60(6.15-19.84)$ & $21.20(11.66-35.62)$ & $14.01(9.39-20.03)$ \\
\hline
\end{tabular}

$63 \%$ respectively. There was no difference in the survival of patients with a more definite laboratory supported diagnosis of myasthenia gravis and the survival of patients which only fulfilled one of the three paraclinical criteria (data not shown).

Figure 1 shows that in both sexes, the survival of patients with myasthenia gravis was shorter than the survival of the corresponding age matched population. The prognosis in women was significantly better than in men $(\mathrm{p}<0.01)$. Women were, however, younger than men (median age for women: 44.3 (range 3-80) years; for men: 62.1 (range 5-85) years; $\mathrm{p}<0.0005)$ ).

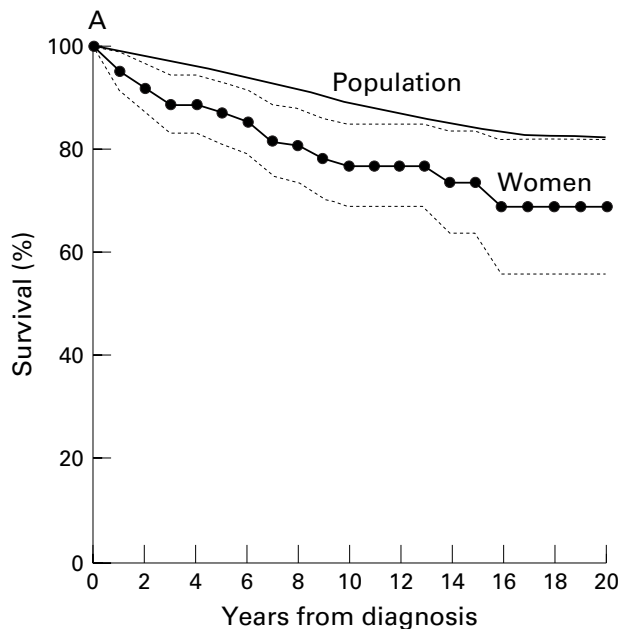

Figure 2 shows that age had a negative influence on survival. The younger patients had a better prognosis than patients aged 60 years or more at the time of diagnosis $(\mathrm{p}<0.0001)$. The survival of the younger patients was not significantly different from the survival of the corresponding age matched Danish population, whereas the survival of the older patients was poorer than expected. There was no difference in survival between old men and old women (data not shown).

Figure 3 shows the survival curves for the 94 thymectomised patients and for the 118 non-thymectomised patients compared with the expected survival of the corresponding age matched Danish population. The three, five, 10 , and 20 year survival rates of the thymectomised patients were $94 \%, 94 \%, 86 \%$, and $79 \%$ respectively. The corresponding figures for the non-thymectomised patients were 78 , 71,56 , and $51 \%$. This difference was highly significant $(\mathrm{p}<0.00001)$. However, the thymectomised patients were younger (median 34.5 years, range 3-75 years) than the nonthymectomised patients (median 65.2 years,

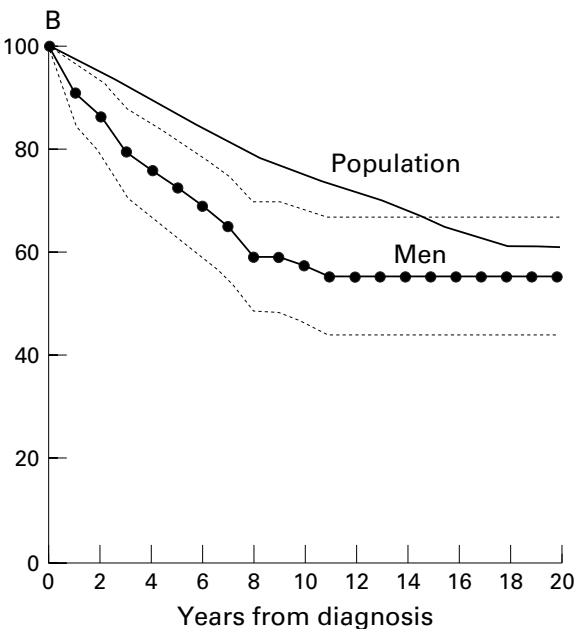

Figure 1 Survival of 212 patients with myasthenia gravis diagnosed 1975-89, western Denmark. Follow up 31 December 1994. (A) women $(n=125)$. (B) men $(n=87)$. Dotted lines $=95 \%$ confidence intervals $(95 \%$ CIs $)$. Solid lines $=$ expected survival of the corresponding Danish population.
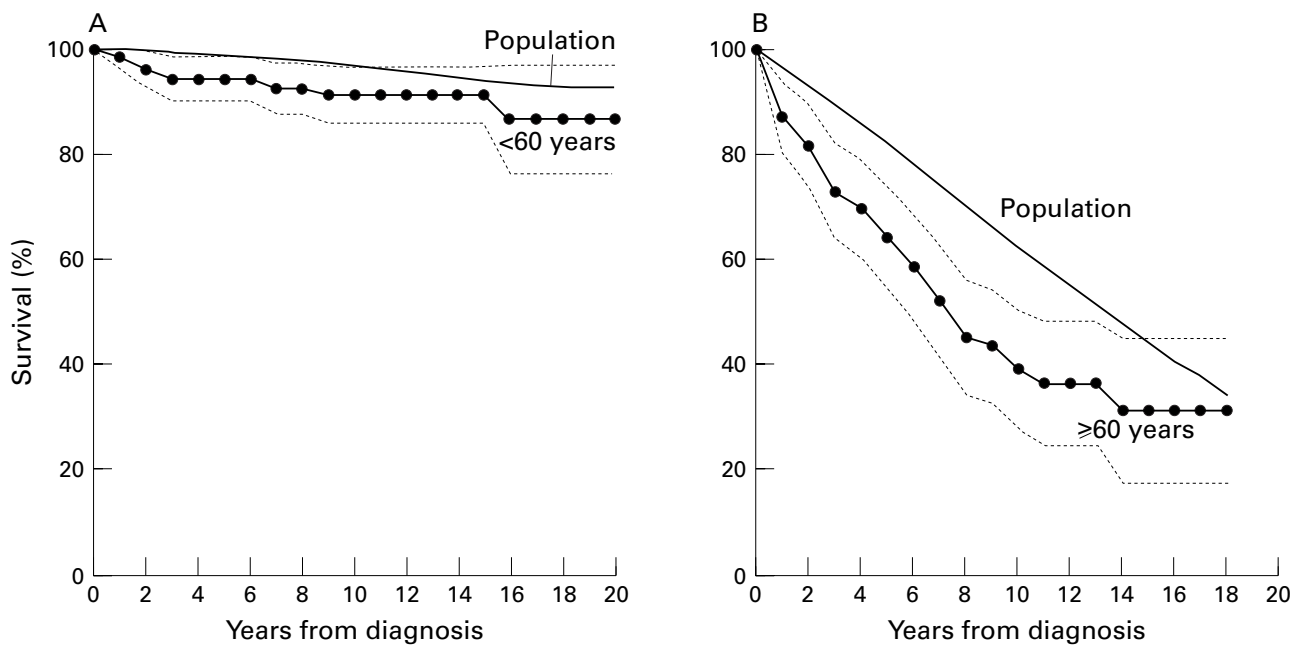

Figure 2 Survival of patients with myasthenia gravis according to age at diagnosis. $(A)<60$ years $(n=121) .(B) \geqslant 60$ years $(n=91)$. Dotted lines $=95 \%$ CIs; solid lines=expected survival of the corresponding Danish population. 

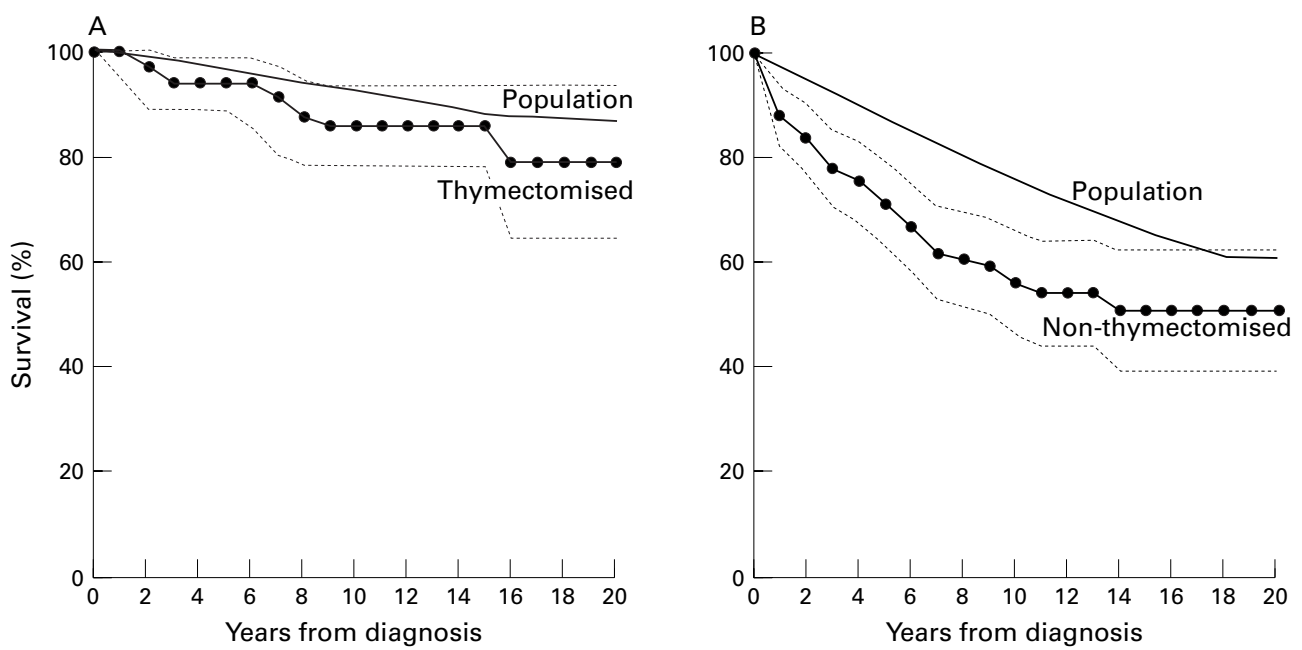

Figure 3 Survival of $(A)$ thymectomised patients with myasthenia gravis $(n=94)$ and $(B)$ non-thymectomised patients $(n=118)$. Dotted lines $=95 \%$ CIs; solid lines $=$ expected survival of the corresponding Danish population.

range $4-85$ years) $(\mathrm{p}<0.00001)$. The survival of the thymectomised patients was not different from what was expected whereas the survival of the non-thymectomised patients was significantly poorer than expected. Of the patients selected for thymectomy, $52 \%$ were classified in group II B, III, or IV (moderate or severe myasthenia gravis) whereas $25 \%$ of the nonoperated patients were in these groups. Forty of the non-thymectomised patients (34\%), but only 12 of the thymectomised patients (13\%) had a serious condition such as cardiovascular disease, cancer, chronic pulmonary disease, or diabetes associated with myasthenia gravis at the time of diagnosis.

The variables (age at diagnosis, sex, thymectomy or not) which were significantly related to survival in the univariate analysis were further analysed in a Cox regression analysis. This disclosed that only age attained significance $(p<0.00001)$, whereas the $p$ values for sex and thymectomy were 0.054 and 0.21 respectively.

Figure 4 shows the survival data subdividing the patients into groups I, II A, II B, and III of the Osserman-Genkins classification (the six patients in group IV did not allow survival calculation). Comparing the overall distribution of the survival curves, the patients classified in group II A had a significantly better survival than the patients in group III $(p<0.05)$. There was no difference in survival between patients in group II B and patients in group III $(p=0.55)$ nor between patients in group II A and II $B(p=0.11)$. Patients in group III showed a rapid decline in survival within the first two to three years from diagnosis. Patients with pure ocular myasthenia gravis (group I) had, as expected, the best survival but only within the first seven years. After this period these patients showed a rapid decline in survival.

Figure 5 shows the survival of the 26 patients with myasthenia gravis with a thymoma. The five and 10 year survival rates were $86 \%$ and $66 \%$ respectively. After this time the rate declined to $33 \%$ at 16 years.

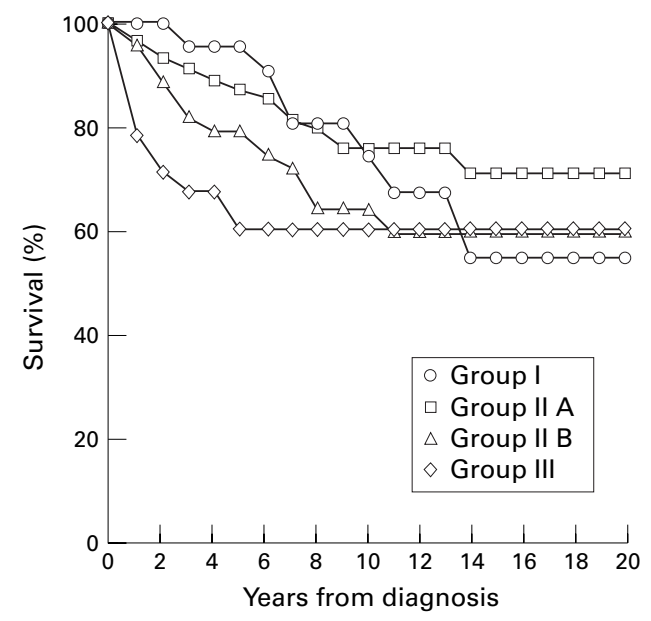

Figure 4 Survival of patients with myasthenia gravis according to the Osserman-Genkins classification.

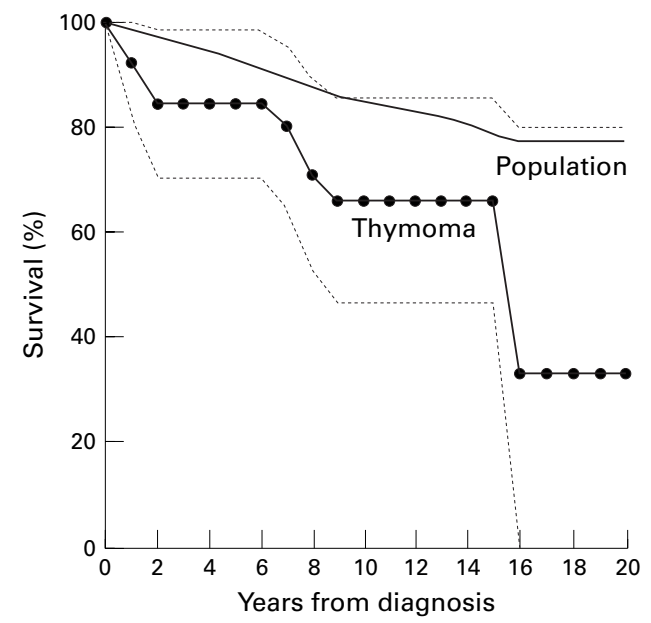

Figure 5 Survival of 26 patients with myasthenia gravis with thymoma. Dotted line $=95 \%$ CIs; solid line $=$ expected survival of the corresponding Danish population.

\section{Discussion}

A precise description of the natural history of a disease requires reliable data based on unselected patient materials. This can only be achieved by using population based data and a well defined end point of outcome such as 
death. Nevertheless, most descriptions of the outcome in myasthenia gravis are based on hospital data using a qualitative assessment of the clinical course of the disease according to the Osserman-Genkins classification. There have been few studies on the survival of patients with myasthenia gravis, ${ }^{10}$ one of which was population based with a sufficient case finding procedure. ${ }^{10}$ The study by Perlo et $a l^{11}$ was based on hospital data from two large neurological departments with a special interest in myasthenia gravis. This study may be biased towards a higher frequency of cases of severe myasthenia gravis. We think that our case finding was complete and unselected as the study involved all hospital departments in the study area, and not only highly specialised neurological departments with a special interest in myasthenia gravis. We also consider the information concerning death to be valid as all deaths including the causes in Denmark are registred centrally in the same registry.

\section{MORTALITY}

The crude mortality rate of myasthenia gravis in western Denmark of 1.8 per million per year is similiar to data reported from other countries $^{12-18}$ where the mortality rates have ranged from 0.7 per million per year in the Merseyside conurbation, United Kingdom ${ }^{12}$ to 2.6 per million in central and western Virginia, United States ${ }^{18}$ and to data from eastern Denmark published recently. ${ }^{10}$

In a recent survey Grob et $a l^{19}$ studied mortality from myasthenia gravis in the period 1940-85 using case:fatality ratios. In the period $1940-57,31 \%$ of their patients with generalised myasthenia gravis died. In the period 1966-85, the fatality ratio was reduced to $7 \%$ as a result of improved management of the disease due to the use of assisted ventilation, thymectomy, plasmapheresis, and immunosuppressant drugs such as corticosteroids and azathioprine. In the present study, the annual average case:fatality ratio was as low as $2 \%-3 \%$, a figure comparable with the $7 \%$ reported by Glob et $a l^{19}$ from their latest study period. By contrast with our constant fatality ratio, we found that the myasthenia gravis mortality rate increased during the study period. A similiar finding has been reported from Norway. ${ }^{15}$ This may be explained by an increasing number of deaths as a result of an increasing incidence of myasthenia gravis during the study period.

Below the age of 50 years, the myasthenia gravis mortality is low. As expected, ${ }^{20}{ }^{21}$ the mortality rate increased with age after 50 years. Similarly to Kurtzke, ${ }^{16}$ we found that the mortality rate in the older age groups increased more rapidly in men than in women.

As in the general population, cardiovascular disease and cancer were the most common causes of death in patients with myasthenia gravis. In $27 \%$ of our patients myasthenia gravis was the underlying cause of death but in only two patients a myasthenic crisis was mentioned specifically on the death certificate. This may reflect the efficiency of modern management, as do the few patients dying of respiratory infections. The figures could, how- ever, be biased, as there is always some uncertainty concerning the information on a death certificate. It should be emphasised that five old patients died of a complication due to long term steroid treatment. Others have noted a high complication rate after steroid treatment, ${ }^{22} 23$ especially in old patients. ${ }^{22}$ These findings favour the use of azathioprine in such patients. ${ }^{22}$

SURVIVAL

The present study shows a relatively good overall prognosis in myasthenia gravis, although the survival of both women and men with myasthenia gravis was shorter than that of the corresponding population. As expected old patients had a less favourable prognosis.

Thymectomy is usually advocated in patients with generalised myasthenia gravis, although no prospective case-control study has proved the superiority of thymectomy as opposed to no surgery. In most studies, the effect of thymectomy is estimated using the rate of remission of the disease, or the rate of improvement, or both. Remission of the disease is well defined in most studies. "Improvement" is less well defined and the term is used to describe a qualitative rather than a quantitative difference. By using death as the end point to determinate the effect of a treatment, any bias using a qualitative scale can be avoided. Despite this, few authors have used survival data to examine the effect of thymectomy. Perlo et $a l^{11}$ used survival data to show that thymectomised patients without a thymoma survived longer than their age matched controls. In agreement with this, our study showed that the survival of the thymectomised patients did not differ from the survival of the normal population, whereas those who had not had thymectomy seemed to have an increased mortality compared with the age matched controls. A Cox regression analysis, however, showed that this apparently beneficial effect of thymectomy was biased by the fact that the patients who underwent thymectomy were significantly younger than those who did not. Furthermore, at the time of diagnosis of myasthenia gravis the non-thymectomised patients had a higher frequency of serious conditions associated with myasthenia gravis than the thymectomised patients. This could explain the high mortality rate and the decision of not performing thymectomy in these patients.

It is of interest to determine whether a qualitative scale, such as the Osserman-Genkins classification, correlates with the survival of patients with myasthenia gravis. Within the first seven to eight years from the diagnosis the distribution of the survival curves correlates with the severity of the disease. As expected, patients classified in group II B and III had a less favourable prognosis. Especially among the patients in group III, the mortality was high for the first two to three years. This finding may point to an aggressive treatment in the early phase of the disease. Patients with pure ocular myasthenia gravis had the best prognosis but only within the first seven years from diagnosis. The decline in the survival rate in these 
patients after seven years may be due to an older population and only few patients at risk after seven years. As a result of this a few deaths lead to a considerable drop in the survival rate.

Previous authors ${ }^{11} 1924$ have reported a less favourable survival in patients with myasthenia gravis with a thymoma than in those without a tumour. It has been suggested that this shows the greater severity and progression and lesser response to therapeutic measures in patients with a thymoma. ${ }^{19}$ In agreement with this, we found a 10 and 16 year survival rate of $66 \%$ and $33 \%$ respectively in our patients with thymoma. In 1960, Perlo et al showed a rapid decline in survival in patients with thymoma with myasthenia gravis within the first year from the diagnosis. ${ }^{11}$ We did not find such a tendency. The reason for this difference is not known. It is possible that more modern principles of intensive care and surgery and earlier identification of thymomas by CT in our study period may play a part.

We thank Dr N Kock-Henriksen, Aalborg, Denmark for statistical advice.

1 Simpson JA. Myasthenia gravis: a new hypothesis. Scott Med f 1960;5:419-36.

2 Engel A. Myasthenia gravis and myasthenic syndromes. Ann Neurol 1984;16:519-34.

3 Drachmann DB. Myasthenia gravis. $N$ Engl $f \mathrm{Med}$ 1994;330:1797-810.

4 Osserman KE. Myasthenia gravis. New York: Grune and Stratton, 1958:80.

5 Oosterhuis HJGH. The natural course of myasthenia gravis: a long-term follow-up study. $\mathcal{F}$ Neurol Neurosurg Psychiatry 1989;52:1121-7.

6 Christensen PB, Jensen TS, Tsiropoulos I, et al. Incidence and prevalence of myasthenia gravis in western Denmark and prevalence of myasthenia gravis in western 1989 . Neurology 1993;43:1779-83.

7 Peto R, Pike MC, Armitage P, et al. Design and analysis of randomized clinical trials requiring prolonged observation of each patient: II. analysis and examples. $\mathrm{Br} \mathcal{7}$ Cancer 1977;35:1-39.

8 Greenwood M. Reports of public health and medical subjects, no 33. Appendix 1: errors of sampling of survivorship tables. London: HM Stationary Office, 1926.

9 Cox DR. Regression models and life tables. Fournal of the Royal Statistical Society Series B 1972;34:187-220.

10 Somnier FE, Keiding N, Paulson OB. Epidemiology of myasthenia gravis in Denmark. A longitudinal and comprehensive population survey. Arch Neurol 1991;48: 733-9.

11 Perlo VP, Poskenzer DC, Schwab RS, Viets HR, Osserman KE, Genkins G. Myasthenia gravis: evaluation of treatment in 1355 patients. Neurology 1966;16:431-42.

12 Pennington GW, Wilson A. Incidence of myasthenia gravis in the Merseyside conurbation. In: Viets HR, ed. Myasthenia gravis. Springfield, IL: Charles C Thomas, 1961:33745.

13 Hokkanen E. Epidemiology of myasthenia gravis in Finland. f Neurol Sci 1969;9:463-78.

14 Gudmundsson KR. The prevalence of some neurological diseases in Iceland. Acta Neurol Scand 1968;44:57-69.

15 Storm-Mathisen A. The epidemiology of myasthenia gravis in Norway. Acta Neurol Scand 1984;70:274-84.

16 Kurtzke JF. Epidemiology of myasthenia gravis. Adv Neurol 1978;19:545-64.

17 Kurland LT, Alter M. Current status of the epidemiology and genetics of myasthenia gravis. In: Viets HR, ed. Myasthenia gravis. Springfield, IL: Charles C Thomas, 1961:307-36.

18 Phillips LH, Torner JC, Anderson MS, Cox GM. The epidemiology of myasthenia gravis in central and western Virginia. Neurology 1992;42:1888-93.

19 Glob D, Arsura EL, Brunner NG, Namba T. The course of myasthenia gravis and therapies affecting outcome: Ann $N$ Y Acad Sci 1987;505:472-99.

20 Giagheddu M, Puggioni G, Sanna G, et al. Epidemiological study of myasthenia gravis in Sardinia, Italy (1958-86). Acta Neurol Scand 1989;79:326-33.

21 Osserman KE, Genkins G. Studies in myasthenia gravis: review of a twenty-year experience in over 1200 patients. Mount Sinai 7 Med 1971;38:497-537.

22 Donaldson DH, Ansher M, Horan S, Rutherford RB, Ringel SP. The relationship of age to outcome in myasthenia gravis. Neurology 1990;40:786-90.

23 Johns TR. Long-term corticosteroid treatment of myasthenia gravis. Ann N Y Acad Sci 1987;505:568-83.

24 Evoli A, Batocchi AP, Provenzano C, Ricci E, et al. Thymectomy in the treatment of myasthenia gravis: report of 247 patients. $\mathcal{F}$ Neurol 1988;235:272-6. 